\title{
Erratum to: Correlations between spatial compatibility effects: are arrows more like locations or words?
}

\author{
James D. Miles • Robert W. Proctor
}

(C) Springer-Verlag 2011

\section{Erratum to: Psychological Research DOI 10.1007/s00426-011-0378-8}

The authors have asked us to clarify and correct the following paragraphs of their article.

Instead of:

Results

\section{Response times}

Responses that were erroneous or longer than $1,500 \mathrm{~ms}$ were removed from the analysis. RTs greater than $1,500 \mathrm{~ms}$ constituted $1.2 \%$ of total trials. Significant main effects were found for the factors of condition, $F(2,90)=9.05, p<0.0005, \mathrm{MSE}=3,457$, and compatibility, $F(1,45)=88.00, p<0.0005$, MSE $=618$, but not their interaction, $F(4,180)<1$. Overall RT was faster in the arrow condition $(M=499 \mathrm{~ms})$ than in the location condition $(M=527 \mathrm{~ms}), F(1,45)=16.88, p<0.0005$, $\mathrm{MSE}=2,159$, and the word condition $(M=534 \mathrm{~ms})$, $F(1,45)=15.80, p<0.0005, \mathrm{MSE}=3,509$. There was no difference in RTs between the location and word conditions, $F(1,45)<1$. The compatibility effect was calculated for each condition as the difference between incompatible and compatible trials and is also shown in

The online version of the original article can be found under doi:10.1007/s00426-011-0378-8.

J. D. Miles $(\bowtie)$

Psychology Department, California State University, Long

Beach, 1250 Bellflower Blvd., Long Beach, CA 90840, USA

e-mail: jmiles@purdue.edu

R. W. Proctor

Department of Psychological Sciences, Purdue University,

West Lafayette, IN 47907-1364, USA
Table 1. Paired-sample $t$ tests showed no difference between the compatibility effect for arrows $(M=32 \mathrm{~ms})$, locations $(M=25 \mathrm{~ms})$ and words $(M=27 \mathrm{~ms}), p \mathrm{~s}>$ 0.22 .

The paragraph should read (changes are highlighted in bold):

Results

Response times

Responses that were erroneous or longer than 1,500 ms were removed from the analysis. RTs greater than $1,500 \mathrm{~ms}$ constituted $1.2 \%$ of total trials. Significant main effects were found for the factors of condition, $F(2,90)=9.05$, $p<0.0005$, MSE $=3,457$, and compatibility, $F(1,45)=$ $88.00, p<0.0005, \mathrm{MSE}=618$, but not their interaction, $F(4,180)<1$. Overall RT was faster in the arrow condition $(M=499 \mathrm{~ms})$ than in the location condition $(M=$ $527 \mathrm{~ms}), F(1,45)=16.88, p<0.0005, \mathrm{MSE}=2,159$, and the word condition $(M=534 \mathrm{~ms}), \quad F(1,45)=$ $15.80, p<0.0005$, MSE $=3,509$. There was no difference in RTs between the location and word conditions, $F(1$, $45)<1$. The compatibility effect was calculated for each condition as the difference between incompatible and compatible trials. Paired-sample $t$ tests showed no difference between the compatibility effect for arrows $(M=32 \mathrm{~ms})$, locations $(M=25 \mathrm{~ms})$ and words $(M=$ $27 \mathrm{~ms}), \mathrm{ps}>0.22$.

Instead of:

\section{Correlations}

Pearson's correlations between the overall RT compatibility effect for each stimulus mode showed no significant correlations. As mentioned in the "Introduction", changes 
in the compatibility effect across RT bins may indicate changes in the cognitive processes involved in the compatibility effect across time. Thus, it is possible that although no relation was found between compatibility effects at a global level, compatibility effects at specific times may be related across stimulus modes. Therefore, correlations were conducted between each RT bin for each stimulus mode. Due to the large number of comparisons, a Bartlett's test of sphericity was first run on the resulting correlation matrix and found that the variables were interrelated, $\chi^{2}(36)=155, p<0.0005 .{ }^{1}$ The correlation matrix is shown in Table 2. To summarize, significant correlations were apparent between RT bins within each stimulus mode. This correlation was especially evident between successive bins, with $r \mathrm{~s}>0.55$ and $p \mathrm{~s}<0.01$ for the early-middle and middle-late RT bin comparisons. Critical to the current study, there was neither an observed relation between location RT bins and word RT bins, nor between location RT bins and arrow RT bins. However, all correlations between arrows and words for early and middle RT bins were significant $(r>0.34, p<0.05)$.

The paragraph should read (changes are highlighted in bold):

\section{Correlations}

Pearson's correlations between the overall RT compatibility effect for each stimulus mode showed no significant correlations. As mentioned in the "Introduction", changes in the compatibility effect across RT bins may indicate changes in the cognitive processes involved in the compatibility effect across time. Thus, it is possible that although no relation was found between compatibility effects at a global level, compatibility effects at specific times may be related across stimulus modes. Therefore, correlations were conducted between each RT bin for each stimulus mode. Due to the large number of comparisons, a Bartlett's test of sphericity was first run on the resulting correlation matrix and found that the variables were interrelated, $\chi^{2}(36)=155, p<0.0005 .{ }^{1}$ The correlation matrix is shown in Table 1. To summarize, significant correlations were apparent between RT bins within each stimulus mode. This correlation was especially evident between successive bins, with $r \mathrm{~s}>0.55$ and $p \mathrm{~s}<0.01$ for the early-middle and middle-late RT bin comparisons. Critical to the current study, there was neither an observed relation between location RT bins and word RT bins, nor between location RT bins and arrow RT bins. However, all correlations between arrows and words for early and middle RT bins were significant $(r>0.34, p<0.05)$.

The publisher apologises for any inconvenience caused by these mistakes. 